\title{
FORMACIÓN DE FUTUROS MAESTROS DESDE LA PRÁCTICA EN EL APRENDIZAJE DE LAS MATEMÁTICAS
}

\section{Ma Isabel Baena González, Encarnación Chica Merino,

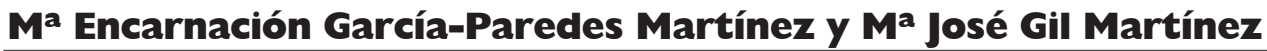

\section{RESUMEN}

El desarrollo de las competencias en el marco del Espacio Europeo de Educación Superior es un punto clave para la adquisición de una preparación académica así como de una preparación profesional en los alumnos universitarios.

Desde este enfoque nos planteamos una experiencia de colaboración entre el Centro de Magisterio "Virgen de Europa" y un centro escolar de Primaria, con el objetivo de favorecer la formación de futuros maestros en el aprendizaje de las matemáticas desde la práctica, así como la colaboración con dicho centro. En esta experiencia nos centramos en la competencia matemática, una de las que obtienen los más bajos rendimientos académicos y donde se mantienen las actitudes más negativas.

Palabras clave: competencia matemática, aprendizaje desde la práctica, resultados de aprendizaje, formación de maestros.

\section{TITLE: TRAINING OF FUTURE TEACHERS THROUGH PRACTICE IN LEARNING MATHEMATICS}

\section{ABSTRACT}

The development of competences in the context of the European Higher Education Area is one of the key points for the acquisition of both academic training and professional training of university students. From this approach we propose a collaborative experience between "Virgen de Europa" Teaching Centre and a Primary school, with the aim of assisting in the training of future teachers through learning mathematics from practice, and also collaborating with the aforementioned centre.

In this experience we focus our attention on mathematical competence, one of those with lowest academic performance and where the most negative attitudes are found.

Keywords: mathematical competence, learning through practice, learning outcome, teacher training.

Correspondencia con las autoras: Ma Isabel Baena González < maribel.baena@magisteriolalinea.com>. Encarnación Chica Merino <echica@magisteriolalinea.com>. Ma Encarnación García-Paredes Martínez <enca.garciaparedes@ magisteriolalinea.com>. Ma José Gil Martínez < mariajose.gil@magisteriolalinea.com>. Centro de Magisterio V. Europa (adscrito a la Universidad de Cádiz). Original recibido: 26-09-13. Original aceptado: 09-01-14 


\section{Introducción}

Es evidente el cambio que ha sufrido el modelo de enseñanza en los últimos tres siglos. Mientras que el objetivo de la enseñanza en el siglo XIX era conseguir la alfabetización del mayor número de ciudadanos, en el siglo $X X$ la educación estaba al alcance de toda una población y los estudios universitarios para una gran parte de ella. Actualmente los avances en la información, la comunicación y la tecnología han influido en la educación del siglo XXI convirtiéndola en una educación para todos y a lo largo de toda la vida (long, life, learning; LLL). Este aprendizaje vitalicio constituye un elemento esencial para alcanzar una mayor competitividad, mejorar el rendimiento profesional, adaptarse a los cambios sociales que se vayan produciendo e ir conociéndolos para alcanzar paulatinamente una calidad de vida. En este entorno nace la nueva Universidad Europea donde los futuros titulados universitarios se preparan para desarrollar y adquirir una serie de competencias transversales, genéricas y específicas necesarias, y enfrentarse a un mercado laboral muy competitivo.

Como sabemos, la Declaración de Bolonia (1999) insta a los estados miembros de la Unión Europea a desarrollar e implantar en las nuevas titulaciones unas competencias genéricas o transversales comunes a todos los grados, que deberán desarrollarse potencialmente en todos los estudios, con el fin de dar el máximo de garantías de formación al egresado, bien para continuar su carrera universitaria o bien para incorporarse al mundo laboral. "Se definen como habilidades necesarias para ejercer cualquier profesión de un modo eficaz y productivo. Las competencias específicas son diferentes entre todas las titulaciones. Se trata de competencias que caracterizan a una profesión, son las que en último término llevan a la formación concreta para la que habilita cada grado y, con ello, son las que se exigirán para el desempeño específico de cada profesión". Los graduados en los nuevos estudios deberán ser capaces de enfrentarse a los retos de la sociedad que les ha tocado vivir gracias al dominio de todas y cada una de las competencias asociadas a su grado (Montero, 20I0).

Actualmente se ha acuñado el término "aprendizaje basado en competencias" $(A B C)$ que consiste en establecer las competencias que se consideran necesarias en el mundo actual y que no pueden ser únicamente determinadas por las universidades sin la consulta y participación de las entidades laborales y profesionales. Según Villa y Poblete (2008) el ABC consiste en desarrollar las competencias genéricas o transversales (instrumentales, interpersonales y 
sistémicas) necesarias y las competencias específicas (propias de cada profesión), de las que se ha hablado anteriormente, con el propósito de capacitar a la persona sobre los conocimientos científicos y técnicos, su capacidad de aplicarlos en contextos diversos y complejos, integrándolos con sus propias actitudes y valores en un modo propio de actuar personal y profesionalmente. Es un enfoque de enseñanza-aprendizaje que requiere, por una parte, partir de un perfil académico-profesional que recoja los conocimientos y competencias que se desea desarrollen los estudiantes que estén realizando un determinado tipo de estudios $y$, por otra, se fundamenta en un sistema que progresivamente va desarrollando la autonomía de los estudiantes y su capacidad de aprender a aprender. EI $A B C$ es un enfoque que se entronca bien con el concepto de European Credit Transfer Sistem (ECTS), en el que cada estudiante debe tener la dedicación adecuada para adquirir o desarrollar las competencias propuestas en el tiempo estimado para ello. Es un sistema de aprendizaje personal que combina teoría y práctica y que se aleja del sistema anterior basado fundamentalmente en la memorización que permitía el estudio concentrado e intensivo en determinados momentos.

Según Montero (2010) "en esta nueva perspectiva el papel del estudiante se modifica y cobra un significado especial, porque él mismo deberá ser el motor que genere su aprendizaje $y$, también porque no sólo aprenderá dentro de las instituciones superiores, sino que cualquier situación y experiencia educativa podrá acercarle al conocimiento a lo largo de toda su vida". Siguiendo a esta autora, el profesor se ve sometido a una gran reforma: ahora no sólo tendrá que transmitir una serie de contenidos, sino que el enfoque se deberá encaminar a abrir al alumno las puertas a un futuro profesional más amplio, donde será fundamental una enseñanza coordinada, con mayor carga práctica. Queda claro que la finalidad principal de los estudios de Grado de maestro tiene como objetivo una preparación profesional y no sólo la preparación académica, donde se contempla una formación general y una formación complementaria destinadas ambas al ejercicio de la profesión.

\section{Competencia matemática}

En relación a la competencia matemática como soporte que permite comprender y hacer avanzar a cualquier ciencia, Martínez (2008) expresa que "las matemáticas son un poderoso lenguaje universal que se constituye como la principal herramienta 
para abstraer, generalizar y sintetizar. Es el idioma que utilizan la tecnología y la ciencia, y el instrumento que posibilita el desarrollo de las nuevas tecnologías, que se encuentran en la base del desarrollo, permiten el cambio social y facilitan alcanzar mejores niveles de vida para todos".

Sin embargo, esta materia es la que obtiene los peores resultados escolares. Si recordamos el Informe PISA 2009, los alumnos españoles de quince años muestran un rendimiento 15 puntos por debajo del promedio de la OCDE, fijado en 500 puntos. Entre las causas de ello, Martínez (2008) recoge que "el planteamiento de su enseñanza, alejado de las necesidades del niño y ajeno a su forma de construir el conocimiento, desnaturaliza la evolución de los estudiantes y su ubicación en las futuras ramas del saber", y desde el análisis que hace este autor "en los tiempos actuales no ser mínimamente competentes en el campo de la matemática supone perder oportunidades, tomar decisiones equivocadas, no saber interpretar la realidad, apoyar opciones falsas, etc."

Desde la tarea de formación, consideramos urgente instruir a los futuros maestros de primaria, no sólo en la adquisición de competencias básicas, incluida la matemática, que como señala el libro blanco de la ANECA (2005), la competencia, en este caso matemática, se define como, "ser capaz de ayudar a alcanzar a los alumnos los objetivos del área”, y por tanto tener en cuenta el proceso de tutorización y apoyo del alumno. En este concepto de competencia matemática para estudiantes de magisterio, Lupiáñez y Rico (2008) citado por Friz (20l0) definen unas competencias profesionales que deben adquirir los futuros profesores en relación a qué capacidades hay que esperar que los escolares alcancen sobre cada tema de un curso y a las decisiones personales que el futuro profesor toma a la hora de planificar sus clases. Asimismo Llinares (2002) citado por Friz (2010) señala diferentes dimensiones del concepto de competencia matemática como componentes del conocimiento profesional. Entre ellas destaca el conocimiento didáctico general, conocimiento de las matemáticas y de las matemáticas escolares, conocimiento pedagógico específico de las matemáticas y el conocimiento del currículo matemático.

Como se ha mencionado anteriormente, en la formación de maestros es importante diseñar un programa basado en competencias según recoge el EEES, de forma que articulando la teoría con la práctica (Friz y col., 2010), podamos incidir en los procesos de enseñanza y aprendizaje de las matemáticas y las propias competencias profesionales de los futuros docentes. 
Desde nuestra realidad educativa, y en el Grado en Educación Primaria, según se recoge en la Memoria del Título, el proceso de enseñanza-aprendizaje se concentra en tareas de organización, seguimiento y evaluación del aprendizaje de los estudiantes. Y concretamente las materias del módulo de Enseñanza y aprendizaje de las Matemáticas, quedan definidas por una serie de competencias que el futuro maestro debe desarrollar para enfrentarse al contexto educativo actual. En el anexo I se muestran dichas competencias los resultados de aprendizaje específicos de cada asignatura. Y nuestro Centro de Magisterio, de La Línea de la Concepción, ha apostado en estas materias por un trabajo de aprendizaje articulando la práctica y la práctica en el aula, llevando a cabo una experiencia de colaboración con un Centro de Educación Primaria de la misma localidad.

\section{Diseño de la experiencia}

Como se acaba de mencionar, la experiencia de colaboración llevada a cabo entre nuestro centro universitario y un centro de educación primaria de la localidad surge desde un planteamiento de aprendizaje desde la práctica entre ambas entidades. A partir de los resultados de las pruebas de diagnóstico obtenidos por los alumnos de primaria, así como la necesidad de que el alumnado universitario adquiera unas competencias y alcance un perfil de maestro según las necesidades del contexto educativo actual, se vio oportuno diseñar una experiencia con enfoque práctico en el centro escolar de primaria, a través de la cual nuestro alumnado pueda adquirir un aprendizaje de la enseñanza de las matemáticas desde la práctica, al mismo tiempo que se reforzasen contenidos y estrategias matemáticas en el alumnado de primaria del centro colaborador.

En un esfuerzo por encauzar esta línea metodológica de la que se viene hablando, el profesorado del centro universitario llevó a cabo una revisión del currículo y definición de las dimensiones o niveles de dominio de cada una de las competencias en las asignaturas de Matemáticas del Módulo Enseñanza y Aprendizaje de las Matemáticas en el Grado en Educación Primaria, que en los planes de estudio de la Universidad de Cádiz se concretan en las asignaturas: El Conocimiento Matemático en Educación Primaria I, Conocimiento Matemático en Educación Primaria II, Didáctica de las Matemáticas I y Didáctica de las Matemáticas II. En la Tabla I se muestran las competencias definidas en el título y los niveles de dominio propuestos por el equipo docente del centro. 


\section{Competencias específicas \\ Adquirir competencias básicas matemáticas (numéricas, cálculo, geométricas, representaciones espaciales, estimación y medida, organización e interpretación de la información,...).}

Conocer el currículo escolar de matemáticas.

\section{Niveles de dominio}

Conocer y dominar las operaciones matemáticas (numéricas, cálculo, geométricas,...).

Analizar e interpretar enunciados, algoritmos y textos matemáticos.

Aplicar los conocimientos y métodos adquiridos en la resolución de problemas matemáticos.

Conocer y manejar la legislación vigente referida al currículo de Matemáticas en primaria.

Conocer las competencias, contenidos y criterios de evaluación en cada ciclo de primaria.

Analizar y procesar la información.

Analizar, razonar y comunicar propuestas matemáticas.

Razonar y resolver situaciones que presentan una dificultad y requieren deliberar e investigar utilizando los conocimientos matemáticos adquiridos.

Comunicar y saber transmitir.

Resolver problemas y actividades matemáticas relacionadas con la vida cotidiana, expresando

Plantear y resolver problemas vinculados con la vida cotidiana. correctamente los resultados obtenidos y justificando dichos resultados con argumentos matemáticos.

Plantear y diseñar actividades matemáticas relacionadas con la vida cotidiana dirigidas a los alumnos de la etapa de primaria en sus distintos niveles.

Valorar la relación entre matemáticas y ciencias como uno de los pilares del Conocer la historia de las matemáticas y su relación con las ciencias experimentales.

Valorar la incidencia de las matemáticas en la pensamiento científico. interpretación y estudio de fenómenos sociales, culturales y naturales.

Desarrollar y evaluar contenidos del currículo Conocer y actualizar los recursos didácticos en la enseñanza de las matemáticas.

mediante recursos didácticos apropiados y promover las competencias correspondientes en los estudiantes. Aplicar dichos recursos mediante tareas y situaciones didácticas adecuadas en el desarrollo y evaluación de los contenidos curriculares para la adquisición de las competencias.

Tabla I. Competencias específicas (definidas en el Título) y niveles de dominio (planteados por el profesorado del Centro de Magisterio V. Europa) de las asignaturas de matemáticas en la Titulación de Grado en Educación Primaria de la Universidad de Cádiz, (20/2) 
Una vez se solventaron cuestiones de carácter organizativo entre ambos centros, se diseñaron cuatro fases para su desarrollo. En la Figura I se muestran en un esquema las fases de dicha experiencia.

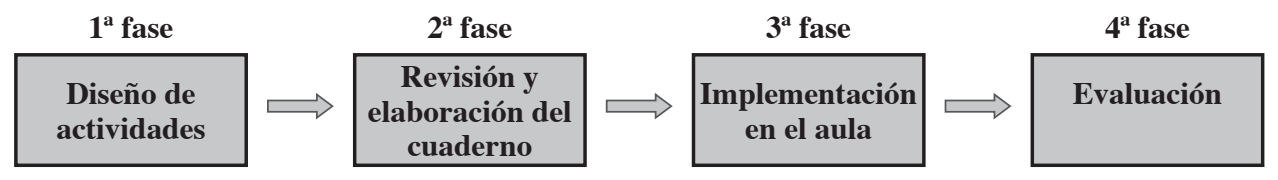

Figura I. Fases de la experiencia de colaboración

En una primera fase, el profesorado de las asignaturas de matemáticas en este título planteó como tarea al alumnado de primer y segundo curso del Grado en Educación Primaria el diseño de actividades específicas para el desarrollo de la competencia matemática en alumnos de segundo ciclo de Primaria. El objetivo de esta fase era que el alumnado universitario se familiarizase con el diseño de este tipo de actividades, que posteriormente deberán realizar en su profesión. Para ello debían tener en cuenta los siguientes criterios:

I. Diseñar las mismas según los contenidos y criterios de evaluación que establece el RD 1513/2006 en Educación Primaria

2. Plantear dichas actividades desde un contexto cercano al alumno.

3. Mantener el formato establecido por la Comunidad Autónoma (Andalucía) para las pruebas de diagnóstico.

En la segunda fase, referida a la "revisión y elaboración del cuaderno" las tareas fueron revisadas por el profesorado universitario una vez finalizadas y se evaluaron siguiendo los criterios antes expuestos.

Posteriormente y con las actividades seleccionadas según estos criterios de evaluación, se elaboró un cuaderno de actividades para facilitar el desarrollo de las mismas en los alumnos del centro de primaria.

La implementación de dichas actividades formó parte de la tercera fase, y se llevó a cabo en las aulas de segundo ciclo de Educación Primaria (segundo nivel) en un centro público de la Línea de la Concepción, con un total de 67 alumnos. El alumnado universitario responsable de realizar las actividades en las aulas de segundo ciclo del 
centro escolar fue el matriculado en las asignaturas de El Conocimiento Matemático en Educación Primaria I y Didáctica de las Matemáticas I, distribuyendo a los alumnos de segundo ciclo en pequeños grupos (entre dos y cuatro alumnos), para favorecer su seguimiento y la resolución de dudas.

Posteriormente, se llevó a cabo la evaluación de la experiencia y para ello, los alumnos de Magisterio realizaron la evaluación de las actividades hechas por los escolares, basándose en los criterios de evaluación que se establecen según la Orden de 28 de junio de 2006 por la que se regulan las pruebas de evaluación de diagnóstico y el procedimiento de aplicación en los centros de Andalucía. El objetivo era situar al futuro maestro en la práctica de la evaluación. También se pidió al alumnado universitario que valorase de forma anónima la experiencia llevada a cabo. Para ello el equipo docente elaboró un cuestionario basándose en las competencias y resultados de aprendizaje de las asignaturas implicadas (Anexo I), y solicitó su opinión acerca de la contribución que la práctica realizada había tenido sobre el desarrollo de esas competencias y resultados de aprendizaje recogidos en el Título de Grado.

\section{Material y método}

El cuestionario elaborado estaba formado por diez ítems construidos en base a los resultados de aprendizaje y dimensiones de la competencia matemática de las asignaturas implicadas del Título de Grado (Tabla 2). Las respuestas se recogían según una escala del I (nada de acuerdo) al 4 (totalmente de acuerdo). Para facilitar la recogida de datos se utilizó la plataforma educativa del Centro.

Dicho cuestionario se administró a una población total de 140 alumnos universitarios del Grado de Educación Primaria, con edades comprendidas entre 18 y 20 años. De ellos, 60 alumnos matriculados en la asignatura El Conocimiento Matemático en Ed. Primaria I, 46 eran mujeres y 14 eran hombres; y 80 alumnos matriculados en Didáctica de las Matemáticas I, 42 eran mujeres y 38 eran hombres (Figuras 2 y 3 ).

El análisis de datos se realizó mediante el paquete estadístico SPSS (v. I5.0) y se obtuvieron estadísticos descriptivos como media, moda, desviación típica, máximos y mínimos. 


\begin{tabular}{|c|c|c|c|c|c|}
\hline \multicolumn{6}{|c|}{$\begin{array}{l}\text { Muestra tu grado de acuerdo entre I (nada de acuerdo) y } \\
4 \text { (totalmente de acuerdo) respecto a las siguientes afirmaciones }\end{array}$} \\
\hline \multicolumn{2}{|r|}{$\begin{array}{l}\text { EL DISEÑO, IMPLEMENTACIÓN Y EVALUACIÓN DE ACTIVIDADES } \\
\text { PARA EL DESARROLLO DE LA COMPETENCIA MATEMÁTICA EN } \\
\left.\text { ALUMNOS DE PRIMARIA ( } 4^{\circ} \mathrm{CURSO}\right), \text { HA CONTRIBUIDO A: }\end{array}$} & I & 2 & 3 & 4 \\
\hline I & Conocer el currículo escolar de matemáticas en primaria & & & & \\
\hline 2 & $\begin{array}{l}\text { Diseñar y plantear problemas vinculados con la vida cotidiana, para } \\
\text { ese nivel }\end{array}$ & & & & \\
\hline 3 & Valorar la relación de las matemáticas con la ciencia & & & & \\
\hline 4 & $\begin{array}{l}\text { Realizar consultas y análisis relativos a los contenidos de } \\
\text { matemáticas en Primaria }\end{array}$ & & & & \\
\hline 5 & $\begin{array}{l}\text { Conocer el papel del bloque numérico, la geometría, la medida y la } \\
\text { estadística en el currículo de matemáticas }\end{array}$ & & & & \\
\hline 6 & Detectar las dificultades que tiene la enseñanza de las matemáticas & & & & \\
\hline 7 & $\begin{array}{l}\text { Constatar los distintos niveles de conocimiento matemático que } \\
\text { tienen los escolares }\end{array}$ & & & & \\
\hline 8 & Tomar contacto con la realidad de un centro escolar & & & & \\
\hline 9 & $\begin{array}{l}\text { Conocer y utilizar la herramienta de evaluación oficial de la Junta } \\
\text { de Andalucía }\end{array}$ & & & & \\
\hline 10 & Ponerme en contacto con la realidad del proceso evaluativo & & & & \\
\hline
\end{tabular}

Tabla 2. Cuestionario para la valoración de la experiencia práctica

Cabe mencionar que el mayor peso de la experiencia en lo que al diseño de las actividades se refiere, fue llevada a cabo por los alumnos de segundo curso, mientras que los de primer curso sólo diseñaron las actividades de un bloque de contenidos de los cuatro que hay en el curriculum de matemáticas y evaluaron los cuadernos de actividades en base a los criterios de evaluación concretados por los alumnos de segundo curso. La implementación en el aula fue llevada a cabo por los alumnos de ambos cursos. 


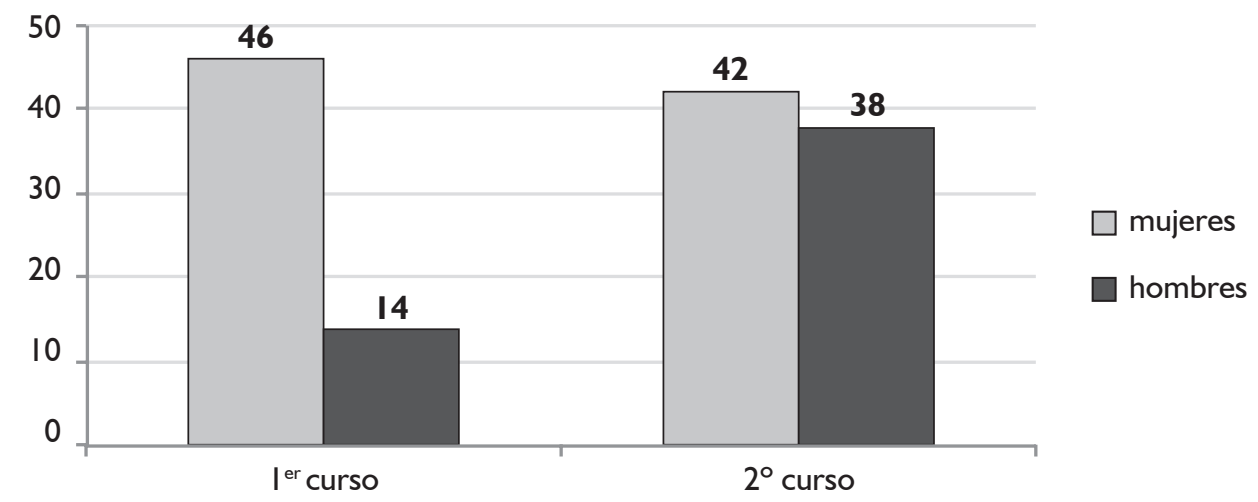

Figura 2. Distribución de mujeres y hombres en las asignaturas de matemáticas de primero y segundo curso del Título en Educación Primaria

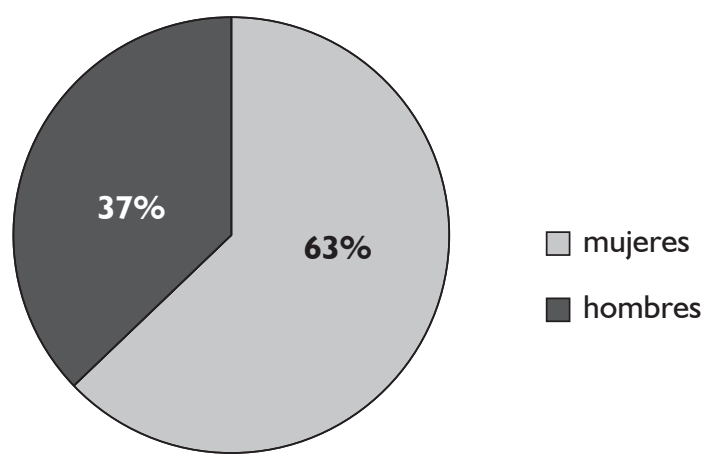

Figura 3. Porcentaje según género en el total de alumnos/as en primero y segundo curso

\section{Resultados}

En el estudio estadístico que se ha realizado para conocer la valoración que los alumnos de Magisterio hacen de la experiencia (Tabla 3) se han analizado los parámetros estadísticos de la muestra total $(\mathrm{N}=140)$, además de la submuestra de primer curso $(\mathrm{N}=60)$ y la submuestra de segundo curso $(\mathrm{N}=80)$. 


\begin{tabular}{|c|c|c|c|c|c|c|}
\hline & \multicolumn{2}{|c|}{ Alumnos $1^{\circ}$} & \multicolumn{2}{|c|}{ Alumnos $2^{\circ}$} & \multicolumn{2}{|c|}{$\begin{array}{l}\text { Muestra } \\
\text { total }\end{array}$} \\
\hline & $\bar{x}_{1}$ & $\sigma_{1}$ & $\bar{x}_{2}$ & $\sigma_{2}$ & $\bar{x}_{\mathbf{T}}$ & $\sigma_{\mathbf{T}}$ \\
\hline $\begin{array}{l}\text { I.Conocer el currículo escolar de } \\
\text { matemáticas en primaria }\end{array}$ & 2,91 & ,74 & 3,11 & ,60 & 3,03 & ,67 \\
\hline $\begin{array}{l}\text { 2. Diseñar y plantear problemas } \\
\text { vinculados con la vida cotidiana, } \\
\text { para ese nivel }\end{array}$ & 3,08 & ,77 & 3,44 &, 57 & 3,29 & 68 \\
\hline $\begin{array}{l}\text { 3. Valorar la relación de las } \\
\text { matemáticas con la ciencia }\end{array}$ & 2,37 & ,69 & $2,4 I$ & ,72 & 2,39 & ,71 \\
\hline $\begin{array}{l}\text { 4. Realizar consultas y análisis } \\
\text { relativos a los contenidos de } \\
\text { matemáticas en primaria }\end{array}$ & 2,73 & ,7I & 2,98 & ,66 & 2,87 & ,69 \\
\hline $\begin{array}{l}\text { 5. Conocer el papel del bloque } \\
\text { numérico, la geometría, la medida } \\
\text { y la estadística en el currículo de } \\
\text { matemáticas }\end{array}$ & 2,93 & ,7I & 3,26 & ,65 & 3,12 & ,69 \\
\hline $\begin{array}{l}\text { 6. Detectar las dificultades } \\
\text { que tiene la enseñanza de las } \\
\text { matemáticas }\end{array}$ & 3,18 & ,75 & 3,25 & ,79 & 3,22 & ,77 \\
\hline $\begin{array}{l}\text { 7. Constatar los distintos niveles } \\
\text { de conocimiento matemático que } \\
\text { tienen los escolares }\end{array}$ & 3,18 & ,83 & 3,11 & ,84 & 3,14 & ,84 \\
\hline $\begin{array}{l}\text { 8. Tomar contacto con la realidad } \\
\text { de un centro escolar }\end{array}$ & 3,55 &, 59 & $3,4 I$ & ,94 & 3,47 & ,81 \\
\hline $\begin{array}{l}\text { 9. Conocer y utilizar la } \\
\text { herramienta de evaluación oficial } \\
\text { de la Junta de Andalucía }\end{array}$ & 2,82 & ,83 & 2,85 & ,81 & 2,84 & ,82 \\
\hline $\begin{array}{l}\text { 10. Ponerme en contacto con la } \\
\text { realidad del proceso evaluativo }\end{array}$ & 3,15 & ,76 & 3,10 & ,84 & 3,12 & ,80 \\
\hline
\end{tabular}

Tabla 3. Resultados de los parámetros estadísticos en la submuestra de $I^{\circ}$ curso $(N=60)$, en la submuestra de $2^{\circ}$ curso $(N=80)$ y en la muestra total $(N=140)$ 
Como puede observarse en los datos expuestos, los aspectos más valorados por el alumnado en la experiencia realizada son los relacionados con la toma de contacto con la realidad de un centro escolar (ítem 8), obteniendo una media de 3,47 en la muestra total y alcanzando valores próximos en la población de primer y segundo curso con medias de 3,55 y 3,4I respectivamente. Creemos que el valor tan alto se ve influenciado por tratarse de alumnos de los primeros cursos, que aún no han realizado prácticas en centros educativos, y esta experiencia de colaboración propicia el contacto con la realidad educativa.

Con valores también altos $(\bar{x}=3,29$ y $\bar{x}=3,22)$ se encuentran los ítems relacionados con el diseño de problemas vinculados con la vida cotidiana (ítem 2) y detectar dificultades que tiene la enseñanza de las matemáticas (ítem 6). Ambos alcanzan valores de medias entre 3,08 y 3,44 en las muestras de primer y segundo curso. La alta valoración de estos aspectos está en coherencia con uno de los criterios establecidos para el diseño de actividades en la primera fase consistente en plantear dichas actividades desde un contexto cercano al alumno.

Asimismo, aspectos relativos a constatar los distintos niveles de conocimiento matemático que tienen los escolares (ítem 7), ponerse en contacto con la realidad del proceso evaluativo (ítem 10), conocer el papel del bloque numérico, la geometría, la medida y la estadística en el currículo de matemáticas (ítem 5) y conocer el currículo escolar de matemáticas en primaria (ítem I) se encuentran con medias entre 3, I4 y 3,03 y están relacionados con competencias profesionales del título de maestro.

Por otro lado el ítem menos valorado fue el 3, que recoge la relación de las matemáticas con la ciencia; tanto en la muestra total $(\bar{x}=2,39)$ como en el grupo de primer curso $(\bar{x}=2,37)$ y en segundo curso $(\bar{x}=2,4 \mathrm{I})$ es el aspecto menos valorado por el alumnado universitario. Aún cuando está vinculado a una de las competencias de las asignaturas (El Conocimiento Matemático en Educación Primaria I y Didáctica de la Matemáticas I), podemos detectar una falta de vinculación de la competencia matemática con las ciencias, por lo que sería conveniente para el futuro, trabajar de forma más intencionada este aspecto del curriculum en el desarrollo de las clases incorporándolo en el diseño de actividades.

También los ítems 9 y 4 obtienen los valores más bajos del conjunto $(\bar{x}=2,84$ y $\bar{x}=2,87$ respectivamente), ambos relacionados con conocer $y$ utilizar las herramientas de evaluación de la Junta de Andalucía y con realizar consultas relativas a los contenidos de matemáticas en primaria. Consideramos que aún cuando son los menos valorados, las puntuaciones obtenidas (sobre 4) indican también una 
valoración positiva relacionada con la primera fase de la experiencia, en la que se solicitaba al alumnado universitario diseñar actividades siguiendo los contenidos y criterios de evaluación que establece el RD 15/3/2006 en Educación Primaria y manteniendo el formato establecido por la Comunidad Autónoma para las pruebas de diagnóstico.

\section{Conclusiones}

Desde el objetivo que nos planteábamos en la introducción de este texto donde se pretende que el futuro maestro de Educación Primaria adquiera unas competencias vinculadas al área de las matemáticas, así como el desarrollo de unas competencias profesionales desde la práctica, se puede concluir, a partir de los datos extraídos del estudio estadístico, que los alumnos lo que más valoran de esta experiencia llevada a cabo durante la docencia de las asignaturas de matemáticas implicadas (centrándonos en la muestra total) son los ítems referidos al contacto directo de nuestros alumnos en las aulas de $4^{\circ}$ de Primaria del centro escolar (ítem 8), el diseño de actividades (ítem 2), y la detección de dificultades que implica la enseñanza de las matemáticas (ítem 6), con unos valores de media que oscilan en la muestra total entre 3,22 y 3,47 . Por lo que podemos afirmar según los resultados, que el alumnado de Grado en Educación Primaria también ve la importancia del objetivo que nos marcábamos al principio de este estudio, su formación desde la práctica como parte integrante y esencial del curriculum de las asignaturas de matemáticas.

Hay que destacar la diferencia en el proceso de formación de los alumnos de primer curso, los cuales están iniciando sus estudios en los contenidos matemáticos, y los de segundo que ya han cursado las dos asignaturas de contenidos matemáticos y están cursando la asignatura de didáctica durante el desarrollo de la experiencia. Es por ello que encontramos diferencias en los valores de las medias de los ítems I y 5 entre el grupo de primero y segundo, relacionados con el manejo del curriculum de matemáticas en Primaria, siendo en ambos casos los valores más altos en los alumnos de $2^{\circ}$ curso. Además el ítem 2 relacionado con el diseño de actividades, también tiene una ligera diferencia entre las dos submuestras. Esto se debe a que en la asignatura de $2^{\circ}$ se trabaja de forma concreta el curriculum de matemáticas de Primaria y el diseño de problemas vinculados con la vida cotidiana, además los alumnos de $2^{\circ}$ curso diseñaron actividades de tres bloques de contenidos diferentes, de geometría, de medida y de estadística, mientras que los alumnos de primer curso diseñaron actividades sólo del bloque de aritmética. 
En general, la valoración de la experiencia según los aspectos recogidos en el cuestionario es alta, lo que confirma la importancia de que en la formación de futuros maestros, los programas articulen teoría y práctica con el objetivo de incidir no sólo en los aprendizajes, sino en el desarrollo de competencias profesionales de los mismos.

Con vistas a la continuación de esta experiencia, como reflejan los resultados del estudio y análisis de la encuesta realizada a estos estudiantes de magisterio, proponemos realizarla en futuros cursos académicos con los alumnos del segundo año con el fin de que estos adquieran los recursos necesarios para su desarrollo, habiendo cursado las dos asignaturas de conocimiento matemático previamente, y teniendo las herramientas suficientes para obtener el máximo rendimiento de ella. Asimismo, poder evaluar también la experiencia desde el centro escolar de primaria donde se lleva a cabo la misma.

\section{Bibliografía}

El Espacio Europeo de la Enseñanza Superior. Declaración conjunta de los Ministros Europeos de Educación (1999). Recuperado el 23/04/2013 de http://www.ond. vlaanderen.be/hogeronderwijs/bologna/links/language/I999_Bologna_Declaration_ Spanish.pdf.

Friz, M., Rodríguez F., Sanhueza, S. y Cardona, M. (2010). Concepciones de los futuros profesores de matemática sobre las competencias profesionales implicadas en la enseñanza de la estadística. CiDd, 470, I-8.

Lupiáñez, J. L. y Rico, L. (2008). Análisis didáctico y formación inicial de profesores: competencias y capacidades en el aprendizaje de los escolares. PNA, 3 (I), 35-48.

Llinares, S. (2002). La práctica de enseñar y aprender a enseñar matemáticas. La generación y uso de instrumentos de la práctica. Revista de Enseñanza Universitaria, 19, II5- 124.

Martínez, J. (2008). Competencias básicas en Matemáticas. Una nueva práctica. Madrid: Wolters Kluwer.

Memoria del título de Grado en Educación Primaria por la Universidad de Cádiz (20/2). Recuperado el I0/06/20I3 de http://www.magisteriolalinea.com/home/ carpeta/pdf/memoria-primaria-final(jun I 3).pdf. 
Ministerio de Educación. Libro Blanco del Título de Grado en Magisterio (2005). Recuperado el 23/04/20I3 de http://www.aneca.es/media/I50404/libroblanco_ jun05_magisteriol.pdf.

Montero, M. (2010). El proceso Bolonia y las nuevas competencias. Tejuelo, 9, 19-37.

Orden de 28 de junio de 2006, por la que se regulan las pruebas de la evaluación de Diagnóstico y el procedimiento de aplicación en los centros de Andalucía. (2006). BOJA.

PISA (2009). Programa para la Evaluación Internacional de los Alumnos. OCDE. Recuperado el 23/04/20I 3 de http://iaqse.caib.es/documents/aval2009- I0/pisa2009informe-espanol.pdf.

Real Decreto 15/3/2006, de 7 de Diciembre por el que se establecen las enseñanzas mínimas de Educación Primaria. (2006). BOE.

Villa, A. y Poblete, M. (2008). Aprendizaje basado en competencias: Una propuesta para la evaluación de las competencias genéricas. Bilbao: Universidad de Deusto. 


\section{Anexo I}

\section{Competencias y resultados de aprendizaje de las asignaturas de matemáticas del primer y segundo curso del Título de Grado en Educación Primaria}

\begin{tabular}{|c|c|}
\hline CURSOI० & Asignatura: EL CONOCIMIENTO MATEMÁTICO EN ED. PRIMARIA I \\
\hline 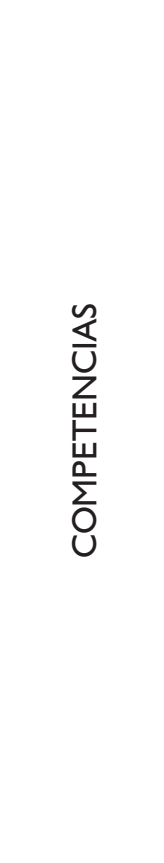 & $\begin{array}{l}\text { CBI } \\
\text { Que los estudiantes hayan demostrado poseer y comprender conocimientos en } \\
\text { un área de estudio que parte de la base de la educación secundaria general, y } \\
\text { se suele encontrar a un nivel que, si bien se apoya en libros de texto avanzados, } \\
\text { incluye también algunos aspectos que implican conocimientos procedentes de la } \\
\text { vanguardia de su campo de estudio } \\
\text { CEI4 } \\
\text { Adquirir competencias matemáticas básicas (numéricas, cálculo, geométricas, } \\
\text { representaciones espaciales, estimación y medida, organización e interpretación } \\
\text { de la información, etc.) } \\
\text { CEI5 } \\
\text { Conocer el currículo escolar de matemáticas general } \\
\text { CEI6 } \\
\text { Analizar, razonar y comunicar propuestas matemáticas } \\
\text { CEI7 } \\
\text { Plantear y resolver problemas vinculados con la vida cotidiana } \\
\text { CEI8 } \\
\text { Valorar la relación entre matemáticas y ciencias como uno de los pilares del } \\
\text { pensamiento científico }\end{array}$ \\
\hline 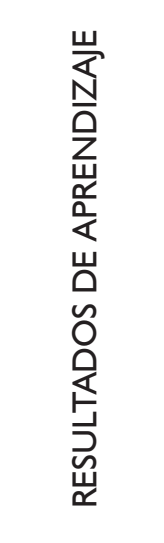 & $\begin{array}{l}\text { I. Adquirir competencias matemáticas a partir de la resolución problemas } \\
\text { 2. Concebir las matemáticas no como un conocimiento cerrado sino como un } \\
\text { proceso en construcción } \\
\text { 3. Valorar el papel de los problemas de la vida cotidiana } \\
\text { 4. Analizar la organización del aula de matemáticas } \\
\text { 5. Conocer el papel de la aritmética en el currículo escolar de las matemáticas } \\
\text { 6. Relativizar el papel de la matemática formal para el aprendizaje } \\
\text { 7. Valorar el papel de la calculadora como generadora de conocimiento } \\
\text { 8. Desarrollar la capacidad de consulta, selección y análisis relativo a las } \\
\text { matemáticas en primaria }\end{array}$ \\
\hline
\end{tabular}




\begin{tabular}{|c|c|}
\hline CURSO2 $^{\circ}$ & Asignatura: EL CONOCIMIENTO MATEMÁTICO EN ED. PRIMARIA II \\
\hline 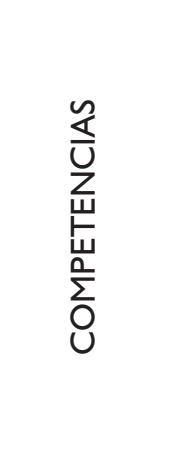 & $\begin{array}{l}\text { CEI4 } \\
\text { Adquirir competencias matemáticas básicas (numéricas, cálculo, geométricas, } \\
\text { representaciones espaciales, estimación y medida, organización e interpretación } \\
\text { de la información, etc.) } \\
\text { CEI5 } \\
\text { Conocer el currículo escolar de matemáticas general } \\
\text { CE } 16 \\
\text { Analizar, razonar y comunicar propuestas matemáticas. } \\
\text { CEI7 } \\
\text { Plantear y resolver problemas vinculados con la vida cotidiana }\end{array}$ \\
\hline 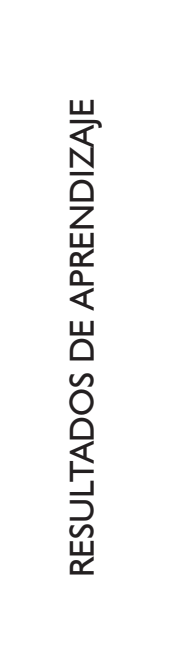 & $\begin{array}{l}\text { I. Adquirir competencias geométricas y métricas a partir de la resolución de } \\
\text { problemas } \\
\text { 2. Conocer el papel de la geometría, la medida y la probabilidad en el currículo } \\
\text { escolar de las matemáticas } \\
\text { 3. Concebir las matemáticas no como un conocimiento cerrado sino como un } \\
\text { proceso en construcción } \\
\text { 4. Conocer las conexiones entre geometría, medida, número y probabilidad } \\
\text { 5. Extraer problemas de la vida cotidiana para plantear } \\
\text { 6. Analizar la organización del aula de matemáticas } \\
\text { 7. Relativizar la importancia de la matemática formal frente a la informal } \\
\text { 8. Valorar el papel de la calculadora como generadora de conocimiento } \\
\text { 9. Desarrollar la capacidad de consulta, selección y análisis de documentos } \\
\text { relativos a las matemáticas en primaria }\end{array}$ \\
\hline
\end{tabular}




\begin{tabular}{|c|c|}
\hline CURSO2 $^{\circ}$ & Asignatura: DIDÁCTICA DE LAS MATEMÁTICAS I \\
\hline 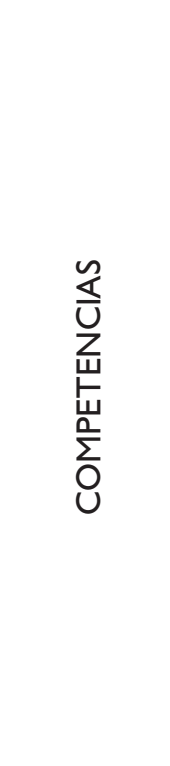 & $\begin{array}{l}\text { CBI } \\
\text { Que los estudiantes hayan demostrado poseer y comprender conocimientos en } \\
\text { un área de estudio que parte de la base de la educación secundaria general, y } \\
\text { se suele encontrar a un nivel que, si bien se apoya en libros de texto avanzados, } \\
\text { incluye también algunos aspectos que implican conocimientos procedentes de la } \\
\text { vanguardia de su campo de estudio } \\
\text { CEI5 } \\
\text { Conocer el currículo escolar de matemáticas general } \\
\text { CE I8 } \\
\text { Valorar la relación entre matemáticas y ciencias como uno de los pilares del } \\
\text { pensamiento científico } \\
\text { CEI9 } \\
\text { Desarrollar y evaluar contenidos del currículo mediante recursos didácticos } \\
\text { apropiados y promover las competencias correspondientes en los estudiantes } \\
\text { CE39 } \\
\text { Conocer y aplicar los procesos de interacción y comunicación en el aula y } \\
\text { dominar las destrezas y habilidades sociales necesarias para fomentar un clima de } \\
\text { aula que facilite el aprendizaje y la convivencia }\end{array}$ \\
\hline 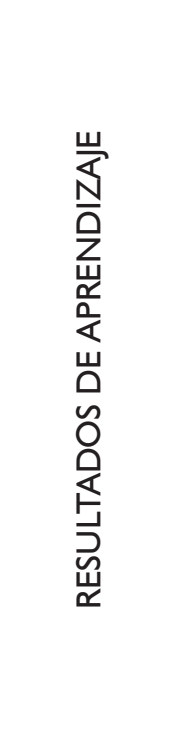 & $\begin{array}{l}\text { I. Comprender elementos de historia de las matemáticas para modificar la visión } \\
\text { de su naturaleza para la enseñanza } \\
\text { 2. Conocer las finalidades de la enseñanza de las matemáticas en la educación } \\
\text { primaria } \\
\text { 3. Conocer la génesis y desarrollo del conocimiento matemático en la educación } \\
\text { primaria } \\
\text { 4. Identificar las dificultades que tiene la enseñanza de las matemáticas } \\
\text { 5. Conocer los fundamentos y el desarrollo del currículo de matemáticas para la } \\
\text { educación primaria } \\
\text { 6. Conocer distintos materiales curriculares en la enseñanza de las matemáticas } \\
\text { 7. Desarrollar la capacidad de consulta, selección, análisis y uso de documentos } \\
\text { relativos a la educación matemática en primaria } \\
\text { 8. Analizar la organización del aula de matemáticas } \\
\text { 9. Ser capaz de gestionar un aula de matemáticas conociendo los aspectos } \\
\text { interactivos que intervienen, facilitando la motivación y permitiendo un } \\
\text { adecuado tratamiento de la diversidad del alumno }\end{array}$ \\
\hline
\end{tabular}

(CB): Competencia básica. (CE): Competencia específica 